Review Article

\title{
Frailty as a predictor of fractures among community-dwelling older people: A systematic review and meta-analysis
}

\section{Gotaro Kojima}

Japan Green Medical Centre, 10 Throgmorton Avenue, London EC2N 2DL, United Kingdom

\section{A R T I C L E I N F O}

\section{Article history:}

Received 18 November 2015

Revised 28 April 2016

Accepted 13 June 2016

Available online 15 June 2016

\section{Keywords:}

Frailty

Fractures

Osteoporosis

Community-dwelling older people

\begin{abstract}
A B S T R A C T
Purpose: To identify prospective studies examining associations between frailty and fractures and to combine the risk measures to synthesize pooled evidence on frailty as a predictor of fractures among community-dwelling older people.

Methods: A systematic literature search was conducted using five databases: Embase, MEDLINE, CINAHL Plus, PsycINFO, and the Cochrane Library for prospective studies on associations between frailty and fracture risk published from 2000 to August 2015 without language restriction. Odds ratios (OR) and hazard ratios (HR) extracted from the studies or calculated from available data were combined to synthesize pooled effect measures using random-effects or fixed-effects models. Heterogeneity, methodological quality, and publication bias were assessed. Meta-regression analyses were performed to explore the cause of high heterogeneity.

Results: Of 1305 studies identified, six studies involving 96,564 older people in the community were included in this review. Frailty and prefrailty were significantly associated with future fractures among five studies with OR (pooled $\mathrm{OR}=1.70,95 \%$ confidence interval $(95 \% \mathrm{CI})=1.34-2.15, \mathrm{p}<0.0001$; pooled $\mathrm{OR}=1.31,95 \% \mathrm{CI}=1.18$ $1.46, \mathrm{p}<0.00001$, respectively) and four studies with $\mathrm{HR}$ (pooled $\mathrm{HR}=1.57,95 \% \mathrm{CI}=1.31-1.89, \mathrm{p}<0.00001$; pooled $\mathrm{HR}=1.30,95 \% \mathrm{Cl}=1.12-1.51, \mathrm{p}=0.0006$, respectively). High heterogeneity was observed among five studies with OR of frailty $\left(\mathrm{I}^{2}=66 \%\right)$. The studies from the United States were found to have a higher fracture risk than from those from other countries in a meta-regression model (regression coefficient $=0.39, \mathrm{p}=0.04$ ) No evidence of publication bias was identified.

Conclusions: This systematic review and meta-analysis showed evidence that frailty and prefrailty are significant predictors of fractures among community-dwelling older people. Treating frailty may potentially lead to lowering fracture risks.
\end{abstract}

(c) 2016 Elsevier Inc. All rights reserved.

\section{Contents}

1. Introduction $\ldots \ldots \ldots, \ldots, \ldots, \ldots$

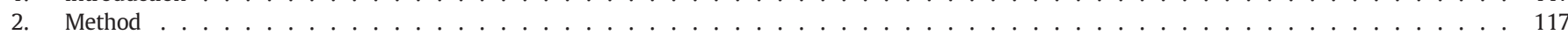

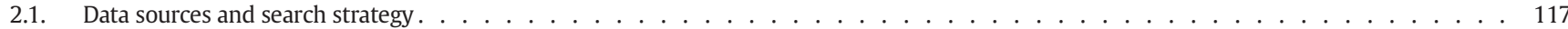

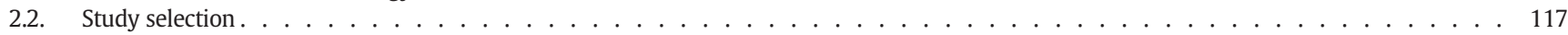

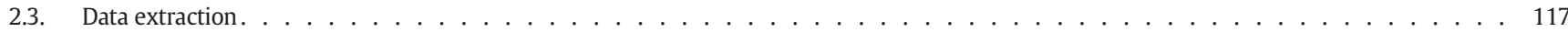

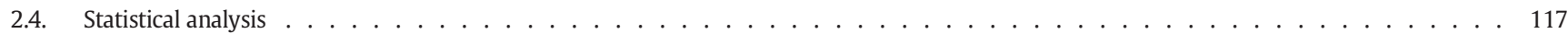

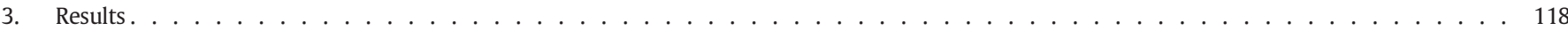

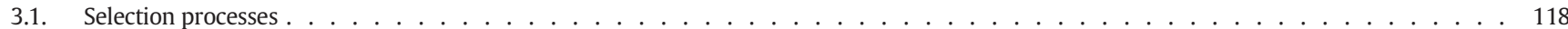

3.2. Study characteristics . . . . . . . . . . . . . . . . . . . . . . . . . . . . . . . 118

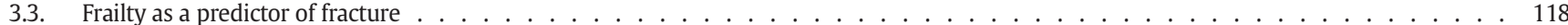

3.3.1. Studies with $\mathrm{OR} \ldots \ldots \ldots \ldots \ldots \ldots \ldots \ldots$

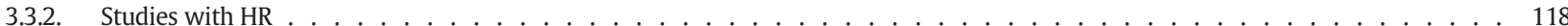

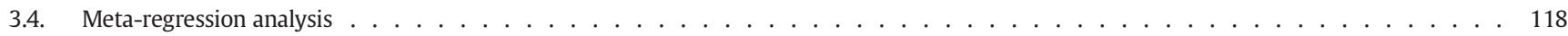

3.5. Publication bias assessment . . . . . . . . . . . . . . . . . . . . . . . . . . . . . . . . . . 119

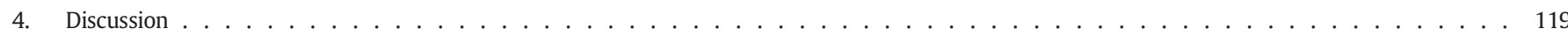

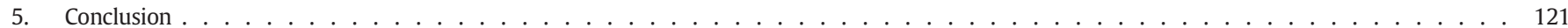

E-mail address: gotarokojima@yahoo.co.jp. 
Conflict of interest . . . . . . . . . . . . . . . . . . . . . . . . . . . . . . . . . . . . . . . . . . . 121

Acknowledgment . . . . . . . . . . . . . . . . . . . . . . . . . . . . . . . . . . . . . . . . . . . . . . . . . . . . . . . 121

References . . . . . . . . . . . . . . . . . . . . . . . . . . . . . . . . . . . . . . . . . . . 121

\section{Introduction}

Fractures are becoming more prevalent as the population ages and the number and proportion of older people grow worldwide [1-3]. Approximately $50 \%$ of women and $20 \%$ of men aged 50 years and older are estimated to have a fracture during the rest of their lives [2]. Fractures can have detrimental impacts physically and mentally on older people and contribute to healthcare burden and costs. In particular, those who sustain hip fractures are often hospitalized for treatment including surgery, which is frequently followed by reduced mobility, functional disabilities, increased dependence, nursing home placement, chronic pain, and high mortality [4]. Fractures have been a major public health concern due to their substantial morbidity and mortality as well as the economic costs.

Osteoporosis is a well-known major risk factor for fragility fractures. Osteoporosis is characterized by low bone mass and microarchitectural deterioration of bone tissue, increasing bone fragility and predisposing older people to an increased fracture risk [5]. Osteoporosis is diagnosed by the presence of fragility fractures or if the bone mineral density of the spine, hip, or wrist is 2.5 standard deviations or more below the reference mean based on the WHO criteria [5].

Frailty, another age-related geriatric syndrome of decreased resistance to stressors and vulnerability to adverse health outcomes due to multisystem impairment [6-9,32,33], shares various risk factors and physiological pathways with osteoporosis, including advanced age, low body weight, low physical activity, sarcopenia, inflammation, and vitamin $\mathrm{D}$ deficiency $[6,10,11]$. Although the relationship between frailty and osteoporosis is not clear, and they may be merely distinct age-related phenomena, some studies showed possible associations [10-12]. Fried et al. defined frailty as having three or more of the five criteria: unintentional weight loss, self-reported exhaustion, weakness, slow walking speed, and low physical activity in the Cardiovascular Health Study (CHS) [13]. Whereas fracture was not examined in this study, several studies have later investigated associations between frailty and fractures and inconsistently shown significant and non-significant results [14-21]. These studies used various frailty criteria and different types of fractures, which makes it difficult to reach the conclusions on frailty as a predictor of fractures. To the best of my knowledge, no systematic review or meta-analysis on associations between frailty and fractures has been conducted. Thus, the objectives of this study were to conduct a systematic search of the literature for prospective cohort studies examining frailty as a predictor of fractures among community-dwelling older people and to perform a meta-analysis to combine the risk measures to synthesize pooled estimates.

\section{Method}

\subsection{Data sources and search strategy}

A literature search was systematically conducted in accordance with a protocol developed within the scope of Preferred Reporting Items for Systematic Review and Meta-Analyses (PRISMA) [22] and Meta-analysis of Observational Studies in Epidemiology (MOOSE) [23] statements. Five electronic databases: Embase, CINAHL Plus, MEDLINE, PsycINFO, and the Cochrane Library, were searched in August 2015 without language restriction for prospective cohort studies examining associations between frailty and a subsequent fracture risk published in 2000 or later. Explosion functions were used if available. The search strategy using Medical Subject Heading (MeSH) terms and keywords was as follows: $\{($ Fractures, bone $(\mathrm{MeSH}))$ OR (Fracture(s) (MeSH)) OR (Fracture $\left.\left.^{*}\right)\right\} A N D$ (Frail elderly (MeSH)) OR (Frailty syndrome $(\mathrm{MeSH}))$ OR (Frailty)\}. Bibliographies of the relevant and included articles were also scrutinized.

\subsection{Study selection}

Studies were included if they involved community-dwelling older people with a mean age of 65 and older, longitudinally examined a risk of any kind of fracture according to baseline frailty status defined by criteria originally designed to measure frailty and validated in population-based studies or its modified versions, and provided odds ratio $(\mathrm{OR})$, risk ratio (RR), or hazard ratio (HR) as a risk measure or data sufficient enough to calculate these measures. Studies were excluded if they defined frailty by disabilities, morbidities, or walking speed; used selected groups of individuals with a certain disease, such as dementia, or hospitalized patients; or were review papers, randomized controlled trials, conference abstracts, letters, comments, or editorials. When the same cohort was used, the study with the largest number of participants was included in this review. When different types of frailty definitions were used, the results of CHS criteria or the largest samples were included.

Studies considered eligible through the title, abstract, and full-text reviews were assessed for quality of methodology using the Newcastle-Ottawa scale for cohort studies [24]. This nine-item checklist was developed to assess the methodological quality of non-randomized studies with three perspectives: (1) the selection of the study groups, (2) the compatibility of the groups, and (3) the ascertainment of either the exposure or outcome of interest for cohort studies [24].

\subsection{Data extraction}

Data extracted were first author, publication year, location (country), sample size, proportion of female participants, age (mean, median, or age criterion for inclusion), frailty criteria, type of fracture, effect measure, and follow-up period.

\subsection{Statistical analysis}

Adjusted, or unadjusted if not available, OR, RR, and HR with a 95\% confidence interval $(95 \% \mathrm{CI})$ of frailty and prefrailty for a fracture risk compared with nonfrailty were extracted from the included studies, or unadjusted OR was calculated using a univariate logistic regression model from the numbers of participants and those who had fractures during the follow-up by frailty status presented in the studies. The heterogeneity among the included studies was assessed using Cochran's Q statistic, and the magnitude of the heterogeneity was assessed using $\mathrm{I}^{2}$ statistic. $\mathrm{I}^{2}$ values of $25 \%, 50 \%$, and $75 \%$ were considered as low, moderate, and high heterogeneity, respectively [25]. OR and HR were separately combined using the inverse variance method to calculate pooled OR and HR. A random-effects model was used if high heterogeneity was detected, and a fixed-effects model was used if the heterogeneity was low to moderate among the included studies. When high heterogeneity was detected, a random-effects meta-regression analysis was conducted to explore the potential causes. Publication bias was assessed using Begg-Mazumdar's and Egger's tests and also by visually inspecting funnel plots.

Analyses were performed using Review Manager 5 (version 5.2, The Cochrane Collaboration, Copenhagen, Denmark), IBM SPSS Statistics 
(version 22, IBM Corporation, Armonk, NY), Comprehensive Meta-Analysis (version 3.3, Biostat, Englewood, NJ), and StatsDirect (version 2.8, StatsDirect, Cheshire, UK).

\section{Results}

\subsection{Selection processes}

The systematic search of the literature using the five electronic databases identified 1304 studies, and one study was found from another source. Among them, 458 studies were excluded because they were duplicates, and 822 studies were excluded through title and abstract screening, leaving 25 studies for full-text review. Of these studies, 19 were excluded because they were letters, comments, editorials, or conference abstracts ( $n=9)$, used the same cohorts $(n=5)$, used non-validated frailty definitions $(n=2)$, used the Frailty Index without categorizing frailty $(n=2)$, or the full-text was not available $(n=1)$. Six studies were left and reviewed for methodological quality. All of the six studies were considered to have adequate methodological quality (mean number of criteria met $=6.7$, range $=5-8$ ) and were included in this review (Table 1). A flow diagram of the literature search and selection process is shown in Fig. 1.

\subsection{Study characteristics}

Characteristics of the six included studies involving 96,564 community-dwelling older people are summarized in Table 1 [14-19]. Three studies were conducted in the United States (US) [17-19], one study each was from the Netherlands [14] and Italy [16], and one study was from multiple countries [15]. Two large studies involved over 40,000 women [15,19]. The smallest cohort contained 749 men and women [16]. Three studies were female only $[15,18,19]$, one study was male only [17], and two studies were mixed $[14,16]$. Mean or median age was approximately 75 to 76 years old, although two studies only reported the numbers of participants in the age groups [15,19]. Modified versions of CHS criteria were used by four studies [15,17-19], and the Longitudinal Aging Study Amsterdam (LASA) frailty instrument [14] and Coselice Study of Brain Aging frailty index [16] were each used once. Various types of fractures were monitored as outcomes, including any, hip, and non-spine fractures. OR whether unadjusted, adjusted, or calculated were available in five studies [14-18], and adjusted HR were available in four studies [14,17-19]. Follow-up periods varied from 1 year [15] to 9 years [18].

\subsection{Frailty as a predictor of fracture}

\subsubsection{Studies with $O R$}

OR of frailty for fractures were available in five studies [14-18] and were combined to calculate pooled OR using a random-effects model due to high heterogeneity $\left(\mathrm{p}=0.02, \mathrm{I}^{2}=66 \%\right.$ ). Frailty was significantly associated with $70 \%$ increased odds of a fracture risk (pooled OR $=1.70$, $95 \% \mathrm{CI}=1.34-2.15, \mathrm{p}<0.0001$ ) (Fig. 2A). As for prefrailty, OR available in three studies $[15,17,18]$ were pooled using a fixed-effects model because of low heterogeneity $\left(\mathrm{p}=0.36, \mathrm{I}^{2}=1 \%\right.$ ). Prefrailty was significantly associated with a higher fracture risk (pooled OR $=1.31,95 \%$ $\mathrm{CI}=1.18-1.46, \mathrm{p}<0.0001$ ) (Fig. 2B).

\subsubsection{Studies with $H R$}

HR of frailty and prefrailty for fractures were available in four [14, 17-19] and three [17-19] studies, respectively, and were combined using fixed-effects models as heterogeneity was low $\left(\mathrm{I}^{2}=3 \%\right.$ and $0 \%$, respectively). Both frailty and prefrailty were significantly associated with a higher risk of fractures (pooled $\mathrm{HR}=1.57,95 \% \mathrm{CI}=1.31-1.89$, $\mathrm{p}<0.00001$; pooled $\mathrm{HR}=1.30,95 \% \mathrm{CI}=1.12-1.51, \mathrm{p}=0.0006$, respectively) (Fig. 2C).

\subsection{Meta-regression analysis}

A high degree of heterogeneity was observed among OR of frailty for a fracture risk in five studies with OR of frailty [14-18] ( $\left.\mathrm{I}^{2}=66 \%\right)$. Random-effects meta-regression analyses were performed using potential causes of the high heterogeneity as a modulator separately. The modulators used included publication year, location (US vs. non-US), sample size, proportion of female participants (\%), mean or median age (one study not providing the mean or median age was excluded(15)), frailty criteria (physical frailty criteria (eventually only CHS) vs. multidimensional criteria), fracture outcome (hip fracture vs. others), follow-up period (year), and methodological quality score. Among these factors, only the study location was found to be a significant modulator. Fig. 3 is a bubble plot illustrating that the US studies $[17,18]$ showed higher OR for fractures than the non-US studies [14-16] (regression coefficient $=$ 0.39 for the US studies, standard error $=0.12,95 \% \mathrm{CI}=0.01-0.77, \mathrm{p}=$

Table 1

Summary of included studies on frailty and fracture risk among community-dwelling older people.

\begin{tabular}{|c|c|c|c|c|c|c|c|c|c|c|}
\hline Author/Study & Year & Location & Sample size & Female (\%) & Age $^{b}$ & Frailty criteria & Fracture outcome & Effect measure & Follow-up period & NOS \\
\hline $\begin{array}{l}\text { de Vries et al. [14] } \\
\text { LASA }\end{array}$ & 2013 & Netherlands & 1509 & $51.8 \%$ & 75.6 & LASA frailty instrument & Any fracture & $\begin{array}{l}\mathrm{aHR} \\
\mathrm{aOR}\end{array}$ & 6 years & $8 / 9$ \\
\hline $\begin{array}{l}\text { Tom et al. [15] } \\
\text { GLOW }\end{array}$ & 2013 & Multiple $^{\mathrm{a}}$ & 44,072 & $100 \%$ & $\geq 55$ & $\mathrm{mCHS}$ & Any fracture & $\mathrm{aOR}$ & 1 year & $6 / 9$ \\
\hline $\begin{array}{l}\text { Forti et al. [16] } \\
\text { CSBA }\end{array}$ & 2012 & Italy & 749 & $55.4 \%$ & 74.7 & CSBA index & Any fracture & uOR & 4 years & $5 / 9$ \\
\hline $\begin{array}{l}\text { Ensrud et al. [17] } \\
\text { MrOS }\end{array}$ & 2009 & US & 3110 & $0 \%$ & 76.4 & $\mathrm{mCHS}$ & Nonspine fracture & $\begin{array}{l}\text { aHR } \\
\text { cOR }\end{array}$ & 3 years & $5 / 9$ \\
\hline $\begin{array}{l}\text { Ensrud et al. [18] } \\
\text { SOF }\end{array}$ & 2007 & US & 6467 & $100 \%$ & 76.7 & $\mathrm{mCHS}$ & Hip fracture & $\begin{array}{l}\text { aHR } \\
\text { cOR }\end{array}$ & 9 years & $8 / 9$ \\
\hline $\begin{array}{l}\text { Woods } 2005 \text { [19] } \\
\text { WHI-OS }\end{array}$ & 2005 & US & 40,657 & $100 \%$ & $65-79$ & $\mathrm{mCHS}$ & Hip fracture & aHR & 5.9 years & $8 / 9$ \\
\hline
\end{tabular}

aHR: Adjusted hazard ratio.

a/u/cOR: Adjusted/Unadjusted/Calculated odds ratio.

CSBA: Conselice Study of Brain Aging Study.

GLOW: Global Longitudinal Study of Osteoporosis in Women.

LASA: Longitudinal Aging Study Amsterdam frailty instrument.

mCHS: Modified Cardiovascular Health Study frailty index.

MrOS: Osteoporotic Fractures in Men Study.

NOS: Newcastle-Ottawa scale for cohort studies.

SOF: Study of Osteoporotic Fractures Study.

WHI-OS: Women's Health Initiative Observational Study.

a Australia, Belgium, Canada, France, Germany, Italy, Netherlands, Spain, the United Kingdom, the Unites States.

b Mean, median, or age criterion for inclusion. 


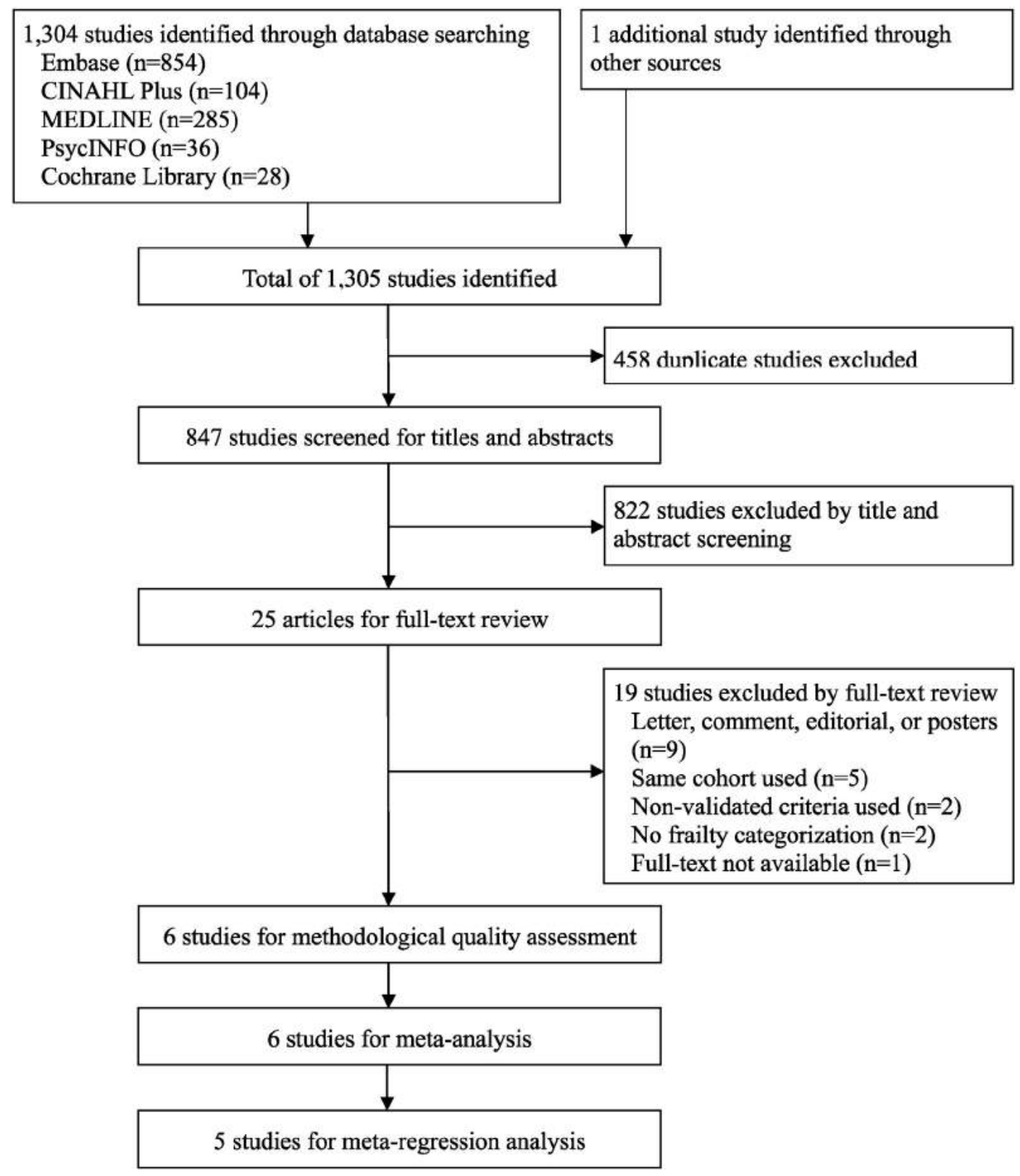

Fig. 1. Flow chart of systematic literature review.

$0.04)$. This model explained $56 \%$ of between-study variance $\left(R^{2}\right.$ ana$\log =0.56)$. Heterogeneity among the three non-US studies [17-19] was low $\left(\mathrm{I}^{2}=0 \%\right)$, and the pooled fracture risk was mildly reduced (pooled $\mathrm{OR}=1.44,95 \% \mathrm{CI}=1.26-1.65, \mathrm{p}<0.00001$ ). However, high heterogeneity remained in the two US studies $[17,18]\left(\mathrm{I}^{2}=72 \%\right)$, and the pooled fracture risk (pooled $\mathrm{OR}=2.01,95 \% \mathrm{CI}=0.163-2.47$, $\mathrm{p}<0.0001$ ) was significantly higher than that of the three non-US studies [14-16] (subgroup difference $\mathrm{p}=0.008$ ).

\subsection{Publication bias assessment}

Publication bias was assessed using Begg-Mazumdar's and Egger's tests among five studies with OR and four studies with HR, and no evidence of publication bias was observed in these two groups (both $\mathrm{p}$ values $>0.05$ ). Asymmetry, which is suggestive of publication bias, was not observed in the funnel plots for four study groups: studies with OR and HR of frailty and prefrailty (Figures not shown).

\section{Discussion}

The pooled data from the six studies involving 96,564 communitydwelling older people suggested that both frailty and prefrailty were significant predictors of fractures. It was suggested that study location may have had an effect on the degree of fracture risks.

No international consensus has been reached regarding a definition of frailty [6]. A wide array of definitions and criteria have been developed and used in clinical and research settings [26]. The CHS criteria are frequently used frailty criteria in the literature, and they were used by four of the six included studies [15,17-19]. The same individuals can be classified differently in terms of frailty by different criteria, and even by the same criteria depending on how they are modified [27], which may potentially lead to different outcomes. Fracture risks based on two different frailty definitions, the CHS criteria and the Study of Osteoporotic Fractures (SOF) frailty index, were compared in the same cohorts by two studies $[17,28]$. The SOF frailty index consists of three components: weight loss, inability to rise from a chair, and 
A

\begin{tabular}{|c|c|c|c|c|c|c|c|c|}
\hline Study or Subgroup & log[Odds Ratio] & SE & Weight & $\begin{array}{c}\text { Odds Ratio } \\
\mathrm{N}, \text { Random, } 95 \% \mathrm{Cl}\end{array}$ & & $\begin{array}{r}\text { Odds } 1 \\
N, \text { Randor }\end{array}$ & $\begin{array}{l}\text { Ratio } \\
\mathrm{m}, 95 \% \mathrm{Cl}\end{array}$ & \\
\hline de Vries 2013 & 0.198851 & 0.193002 & $18.2 \%$ & $1.22[0.84,1.78]$ & & 7 & $\because$ & \\
\hline Tom 2013 & 0.378436 & 0.076407 & $29.9 \%$ & $1.46[1.26,1.70]$ & & & $\rightarrow-$ & \\
\hline Forti 2012 & 0.565314 & 0.295269 & $11.2 \%$ & $1.76[0.99,3.14]$ & & & & \\
\hline Ensrud 2009 & 1.09293 & 0.233721 & $15.0 \%$ & $2.98[1.89,4.72]$ & & & & \\
\hline Ensrud 2007 & 0.597187 & 0.11839 & $25.6 \%$ & $1.82[1.44,2.29]$ & & & & \\
\hline Total $(95 \% \mathrm{Cl})$ & & & $100.0 \%$ & $1.70[1.34,2.15]$ & & & & \\
\hline \multicolumn{5}{|c|}{$\begin{array}{l}\text { Heterogeneity: } \mathrm{Tau}^{2}=0.04 ; \mathrm{Chi}^{2}=11.77, \mathrm{df}=4(\mathrm{P}=0.02) ; \mathrm{I}^{2}=66 \% \\
\text { Test for overall effect: } Z=4.38(P<0.0001)\end{array}$} & 0.2 & $\begin{array}{c}0.5 \\
\text { Decreased Risk }\end{array}$ & Increasec & 5 \\
\hline
\end{tabular}

B

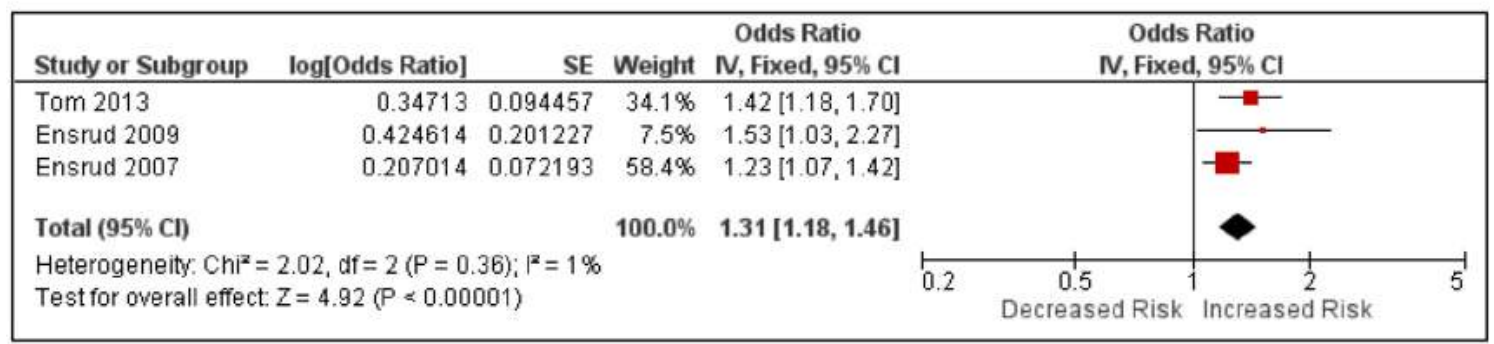

C

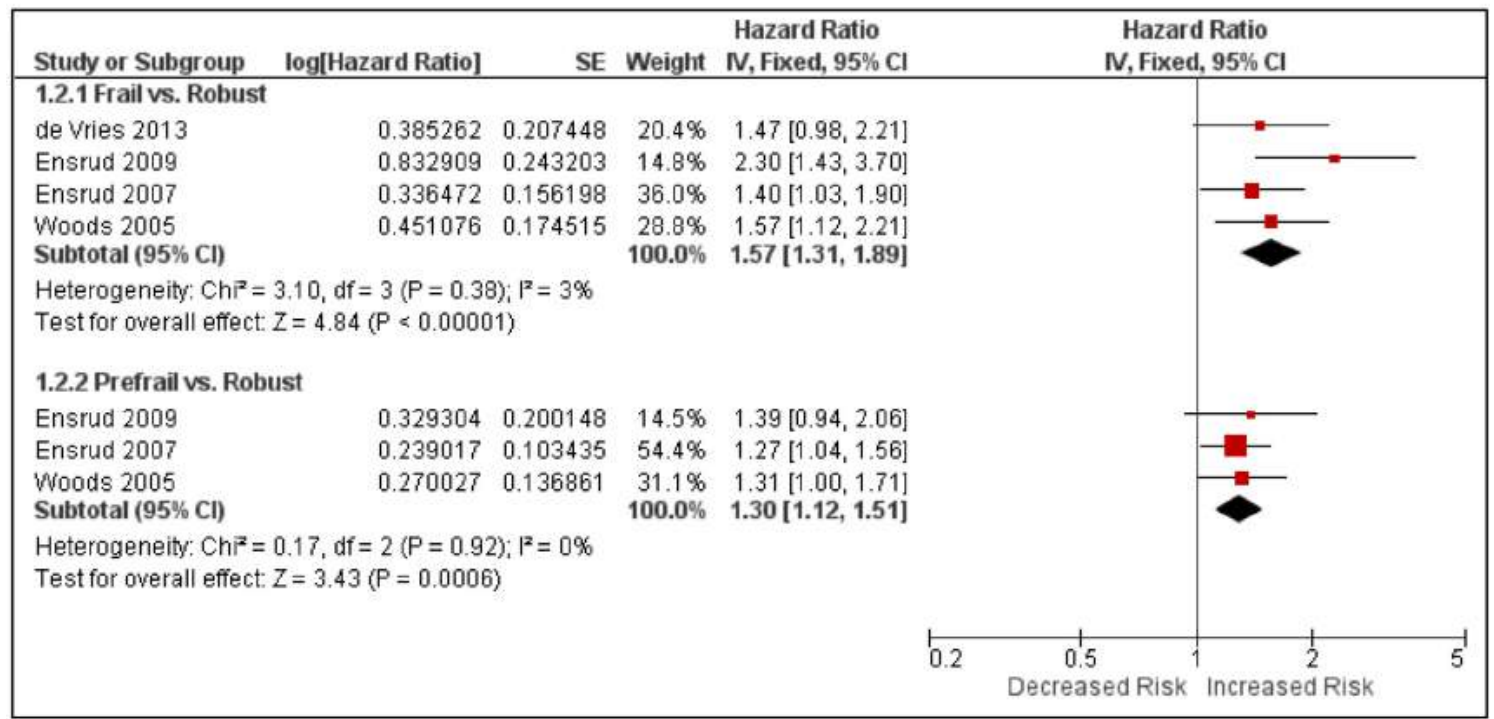

Fig. 2. Forest plots of fracture risk by frailty. Studies presenting odds ratios for frailty (A) and prefrailty (B) and studies presenting hazard ratios for frailty and prefrailty (C).

poor energy, and frailty is defined by the presence of any two of them. Presumably because of the similarity between the CHS and SOF criteria, fracture risks according to frailty based on both criteria were comparable in these studies ( $\mathrm{HR}=2.30$ by CHS criteria vs. $\mathrm{HR}=2.15$ by SOF criteria(17); HR $=1.71$ by CHS criteria vs. HR $=1.79$ by SOF criteria [28]). On the other hand, another study demonstrated a considerable difference in findings based on two frailty criteria [16]. The Conselice Study of Brain Aging Study (CSBA) index defines frailty as having three of more of nine components, and classified 30.0\% (225/749) of the cohort consisting of Italian older people aged 65 years and older, while modified SOF criteria identified only 8.1\% (60/741) as frail in the same cohort [16]. Individuals classified frail by the CSBA index had $76 \%$ increased odds for a fracture risk compared with the non-frail ( $\mathrm{OR}=1.76,95 \% \mathrm{CI}=0.99-3.15)$, while those classified frail by the modified SOF criteria had an almost six times higher fracture risk
( $\mathrm{OR}=5.79,95 \% \mathrm{CI}=2.90-11.55)$ than those who were non-frail. In this context, the use of different frailty criteria can be a cause of heterogeneous outcomes.

The high heterogeneity observed in the five studies with OR [14-18] was explored using random-effects meta-regression models, and the study location (US vs. non-US) was found to be a significant modulator in the association between frailty and fractures. The aforementioned use of frailty criteria (physical frailty criteria vs. multidimensional criteria) was also examined, but it did not show any significant association. The fracture risk according to frailty was significantly higher among the US studies than the non-US studies. The possible explanation for the disparity would be a difference in study design. Both of the US studies were originally designed for fracture and employed frequent fracture monitoring systems at a 4-month interval, while the non-US studies were not specifically designed for fractures $[14,16]$ or monitored 


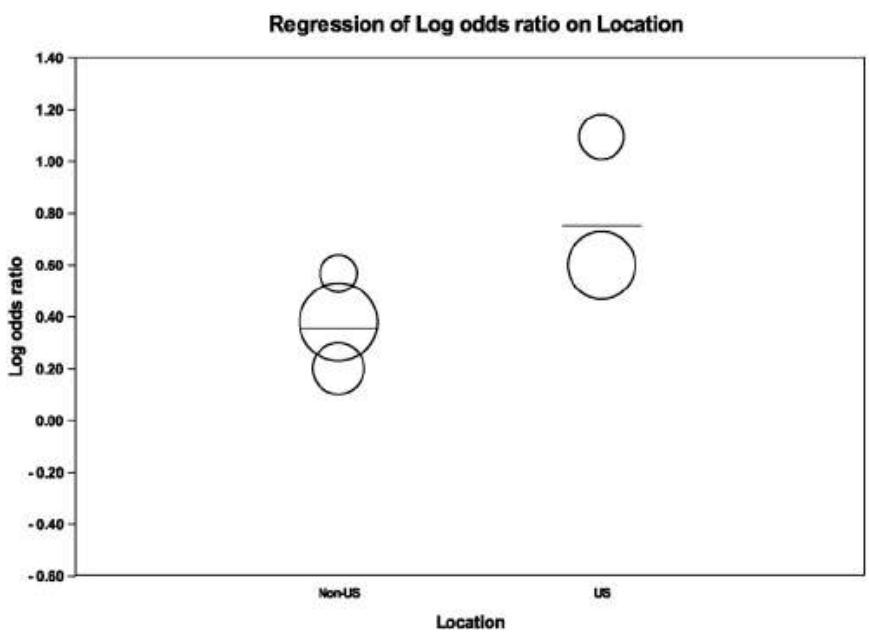

Fig. 3. Bobble plot for association between odds ratio of frailty for fracture risk and study location (US vs. non-US).

fractures at a longer interval of 12 months [15]. These differences may have possibly contributed to underestimating fracture risks in the non-US studies.

Some studies described frailty using the Frailty Index, an accumulated health deficit model, and examined fracture risk according to graded frailty status $[20,21]$. These studies could not be incorporated in the meta-analysis only because they did not dichotomize (frailty and nonfrailty) or trichotomize (frailty, prefrailty, and non-frailty) frailty status. They showed that a higher degree of frailty was significantly associated with a higher risk of fracture, which is consistent with the results of the current meta-analyses.

The findings of this study should be interpreted with caution. Although the search strategy was extensive and reproducible using five databases and comprehensive search terms, there is a possibility that relevant studies were missed or misclassified, as all processes were conducted by one investigator. Adjusted OR were not available in three studies: one study provided only unadjusted OR and two studies presented data from which unadjusted OR were calculated, while the other studies provided adjusted OR or HR. Adjusted risk measures would be preferable in order to avoid confounding effects of covariates when synthesizing pooled estimates.

One of multiple strengths this study has would be that this is the first systematic review and meta-analysis to demonstrate the associations between frailty and fracture risk among community-dwelling older people. Another strength to be noted is the comprehensive methodology, including an extensive systematic review using five electronic databases and assessments of methodological quality, heterogeneity, and publication bias among the included studies. Moreover, meta-regression analyses were performed to explore possible causes of the high heterogeneity.

The exact mechanisms underlying the association between frailty and a higher risk of fracture are not clear. Considering multidimensional features of frailty and multiple risk factors for falling, the association may be complex and multifactorial. Since falls are one of the common causes of fracture, a higher fracture risk may be attributed to a higher risk of falls according to frailty $[7,10]$. Multiple studies have shown that fall frequency and characteristics were more strongly correlated with fractures than bone mineral density [10].

Another possible explanation is weight loss, which is one of the main components to conceptualize frailty in the CHS and other criteria. Body weight correlated positively with the bone mineral density of the proximal femur in postmenopausal women aged 75 years and older [29]. Weight loss is shown to be a risk factor of hip fracture in a recent systematic review and meta-analysis paper [30]. Among the six included studies, five used the CHS criteria or LASA frailty instrument, both of which contain weight loss as a criterion.

\section{Conclusion}

This systematic review and meta-analysis provide evidence that frailty is a significant predictor of future fractures among communitydwelling older people. Given that frailty and prefrailty can be reversed or improved by interventions [6,31], treating frailty and prefrailty may lead to lowering fracture risks.

\section{Conflict of interest}

None.

\section{Acknowledgment}

This research received no specific financial support from any funding agency in the public, commercial, or not-for-profit sectors.

\section{References}

[1] S.R. Cummings, L.J. Melton, Epidemiology and outcomes of osteoporotic fractures, Lancet (London, England) 359 (9319) (2002) 1761-1767.

[2] J.P. van den Bergh, T.A. van Geel, P.P. Geusens, Osteoporosis, frailty and fracture: implications for case finding and therapy, Nat. Rev. Rheumatol. 8 (3) (2012) 163-172.

[3] K.E. Ensrud, Epidemiology of fracture risk with advancing age, J. Gerontol. A Biol. Sci. Med. Sci. 68 (10) (2013) 1236-1242.

[4] Centers for Disease Control and Prevention (USA), Hip fractures among older adults, http://www.cdc.gov/homeandrecreationalsafety/falls/adulthipfx.html2015 (Accessed 2 September, 2015).

[5] NIH Consensus Development Panel on Osteoporosis Prevention D, and Therapy, Osteoporosis prevention, diagnosis, and therapy, JAMA 285 (6) (2001) 785-795.

[6] A. Clegg, J. Young, S. Iliffe, et al., Frailty in elderly people, Lancet (London, England) 381 (9868) (2013) 752-762.

[7] G. Kojima, Frailty as a predictor of future falls among community-dwelling older people: a systematic review and meta-analysis, J. Am. Med. Dir. Assoc. 16 (12) (2015) 1027-1033.

[8] G. Kojima, Frailty as a predictor of hospitalisation among community-dwelling older people: a systematic review and meta-analysis, J. Epidemiol. Community Health 70 (7) (2016) $722-729$.

[9] G. Kojima, S. Iliffe, S. Jivraj, et al., Association between frailty and quality of life among community-dwelling older people: a systematic review and meta-analysis, J. Epidemiol. Community Health 70 (7) (2016) 716-721.

[10] Y. Rolland, A. van Kan G, A. Benetos, et al., Frailty, osteoporosis and hip fracture: causes, consequences and therapeutic perspectives, J. Nutr. Health Aging 12 (5) (2008) 335-346.

[11] S.A. Sternberg, R. Levin, S. Dkaidek, et al., Frailty and osteoporosis in older women-a prospective study, Osteoporos. Int. 25 (2) (2014) 763-768.

[12] S.L. Ma, J. Oyler, S. Glavin, et al., Self-reported frailty is associated with low calcaneal bone mineral density in a multiracial population of community-dwelling elderly, Osteoporos. Int. 20 (11) (2009) 1837-1846.

[13] L.P. Fried, C.M. Tangen, J. Walston, et al., Frailty in older adults: evidence for a phenotype, J. Gerontol. A Biol. Sci. Med. Sci. 56 (3) (2001) M146-M156.

[14] O.J. de Vries, G.M. Peeters, P. Lips, et al., Does frailty predict increased risk of falls and fractures? A prospective population-based study, Osteoporos. Int. 24 (9) (2013) 2397-2403.

[15] S.E. Tom, J.D. Adachi, F.A. Anderson Jr., et al., Frailty and fracture, disability, and falls: a multiple country study from the global longitudinal study of osteoporosis in women, J. Am. Geriatr. Soc. 61 (3) (2013) 327-334.

[16] P. Forti, E. Rietti, N. Pisacane, et al., A comparison of frailty indexes for prediction of adverse health outcomes in an elderly cohort, Arch. Gerontol. Geriatr. 54 (1) (2012) $16-20$.

[17] K.E. Ensrud, S.K. Ewing, P.M. Cawthon, et al., A comparison of frailty indexes for the prediction of falls, disability, fractures, and mortality in older men, J. Am. Geriatr. Soc. 57 (3) (2009) 492-498.

[18] K.E. Ensrud, S.K. Ewing, B.C. Taylor, et al., Frailty and risk of falls, fracture, and mortality in older women: the study of osteoporotic fractures, J. Gerontol. A Biol. Sci. Med. Sci. 62 (7) (2007) 744-751.

[19] N.F. Woods, A.Z. LaCroix, S.L. Gray, et al., Frailty: emergence and consequences in women aged 65 and older in the Women's Health Initiative Observational Study, J. Am. Geriatr. Soc. 53 (8) (2005) 1321-1330.

[20] X. Fang, J. Shi, X. Song, et al., Frailty in relation to the risk of falls, fractures, and mortality in older Chinese adults: results from the Beijing Longitudinal Study of Aging, J. Nutr. Health Aging 16 (10) (2012) 903-907.

[21] G. Li, L. Thabane, A. Papaioannou, et al., Comparison between frailty index of deficit accumulation and fracture risk assessment tool (FRAX) in prediction of risk of fractures, Bone 77 (2015) 107-114. 
[22] D. Moher, A. Liberati, J. Tetzlaff, et al., Preferred reporting items for systematic reviews and meta-analyses: the PRISMA statement, BMJ (Clinical Research Ed) 339 (2009), b2535.

[23] D.F. Stroup, J.A. Berlin, S.C. Morton, et al., Meta-analysis of observational studies in epidemiology: a proposal for reporting. Meta-analysis of observational studies in epidemiology (MOOSE) group, JAMA 283 (15) (2000) 2008-2012.

[24] G.A. Wells, D. Shea, D. O'Connell, et al., The Newcastle-Ottawa Scale (NOS) for assessing the quality of nonrandomised studies in meta-analyses, http://www. ohri.ca/programs/clinical_epidemiology/oxford.asp (Accessed 31 August 2015).

[25] J.P. Higgins, S.G. Thompson, J.J. Deeks, et al., Measuring inconsistency in meta-analyses, BMJ (Clinical Research Ed) 327 (7414) (2003) 557-560.

[26] L. Rodriguez-Manas, C. Feart, G. Mann, et al., Searching for an operational definition of frailty: a Delphi method based consensus statement: the frailty operative definition-consensus conference project, J. Gerontol. A Biol. Sci. Med. Sci. 68 (1) (2013) 62-67.

[27] O. Theou, L. Cann, J. Blodgett, et al., Modifications to the frailty phenotype criteria: systematic review of the current literature and investigation of 262 frailty phenotypes in the survey of health, ageing, and retirement in Europe, Ageing Res. Rev. 21 (2015) 78-94.
[28] K.E. Ensrud, S.K. Ewing, B.C. Taylor, et al., Comparison of 2 frailty indexes for prediction of falls, disability, fractures, and death in older women, Arch. Intern. Med. 168 (4) (2008) 382-389.

[29] H. Blain, I. Carriere, F. Favier, et al., Body weight change since menopause and percentage body fat mass are predictors of subsequent bone mineral density change of the proximal femur in women aged 75 years and older: results of a 5 year prospective study, Calcif. Tissue Int. 75 (1) (2004) 32-39.

[30] O.B. Lv, X. Fu, H.M. Jin, et al., The relationship between weight change and risk of hip fracture: meta-analysis of prospective studies, Sci. Rep. 5 (2015) 16030.

[31] J.E. Morley, B. Vellas, G.A. van Kan, et al., Frailty consensus: a call to action, J. Am Med. Dir. Assoc. 14 (6) (2013) 392-397.

[32] G. Kojima, Frailty as a Predictor of Nursing Home Placement among CommunityDwelling Older Adults: A Systematic Review and Meta-analysis, J. Geriatr. Phys. Ther. (2016) in press.

[33] G. Kojima, Y. Taniguchi, S. Iliffe, et al., Frailty as a Predictor of Alzheimer Disease, Vascular Dementia, and All Dementia Among Community-Dwelling Older People: A Systematic Review and Meta-Analysis, J. Am. Med. Dir. Assoc. (2016) in press. 